\title{
Estudo comparativo da infiltração marginal em restaurações de classe $\mathrm{V}$
}

\section{Comparative study of microleakage in class $V$ restorations}

\author{
Débora Gonçalves de FARIAS* \\ Rander Pereira AVELAR* \\ Ana Cristina Barreto BEZERRA**
}

\begin{abstract}
FARIAS, D. G. de; AVELAR, R. P.; BEZERRA, A. C. B. Estudo comparativo da infiltração marginal em restaurações de classe V. Pesqui Odontol Bras, v. 16, n. 1, p. 83-88, jan./mar. 2002.

Este estudo teve como objetivo avaliar o comportamento laboratorial de adesivos dentinários, cimentos ionoméricos modificados por resina e resinas compostas modificadas por poliácidos, no selamento das margens gengivais de cavidades de classe V preparadas na junção amelocementária. Quarenta cavidades foram executadas nas faces vestibular e palatina/lingual de 20 terceiros molares humanos extraídos, e restauradas com os seguintes materiais: grupo 1 (G1)- Vitremer (3M); grupo 2 (G2)- Vitremer (3M) e Syntac Sprint/Tetric Ceram (Vivadent); grupo 3 (G3)- Syntac Sprint/Tetric Ceram (Vivadent); grupo 4 (G4)- Prime \& Bond 2.1/Variglass (Dentsply). Após termociclagem em corante azul de metileno, os espécimes foram seccionados longitudinalmente e analisados em lupa estereoscópica para avaliação do grau de infiltração marginal. Os resultados após teste estatístico de Mann-Whitney revelaram melhor vedamento marginal para o grupo 1, quando comparado com o grupo $4(\mathrm{p}=0,05)$, mas não foram demonstradas diferenças significantes entre os demais grupos. Nas condições desse experimento, o material Vitremer ofereceu melhor vedamento marginal que o sistema Prime \& Bond 2.1/Variglass.
\end{abstract}

UNITERMOS: Infiltração dentária; Adesivos dentinários; Cimentos de ionômero de vidro; Resinas compostas.

\section{INTRODUÇÃO}

Inúmeros são os benefícios advindos de uma efetiva união dos materiais restauradores aos tecidos dentários. O preparo cavitário pode ser mais conservador, a descoloração marginal é reduzida, bem como a infiltração marginal e o risco de injúrias pulpares ${ }^{14}$.

Atualmente, as resinas compostas em conjunto com os adesivos dentinários, cimentos de ionômero de vidro modificados por resina e resinas compostas modificadas por poliácidos ocupam um lugar de destaque na Odontologia em função das propriedades adesivas que apresentam. Entretanto, se a união ao esmalte é uma prática facilmente obtida e de sucesso, a união à dentina/cemento é mais dificil e de longevidade ainda questionável $1^{3,14}$.

A grande falha das restaurações estéticas continua centrada nas margens cervicais localizadas em dentina ou cemento, onde a adesividade é mais dificil de ser obtida devido à complexa estrutura dentinária e à contínua exsudação de fluidos advindos da polpa ${ }^{1}$.

$\mathrm{O}$ vedamento das margens cavitárias localizadas em dentina/cemento utilizando materiais fotoativados também é comprometido pela contração de polimerização que sofrem. A força de união dos sistemas adesivos, cimentos de ionômero de vidro modificados por resinas e resinas compostas modificadas por poliácidos é insuficiente diante da contração de polimerização, o que provoca um "afastamento" da restauração na região gengival e "gaps" que permitem a penetração de fluidos e bactérias ${ }^{2}$.

Atualmente, os materiais estéticos constituem a melhor opção para restaurações classe V, mas o grande número de produtos disponíveis no mercado e sua curta permanência muitas vezes dificulta uma avaliação clinica a longo prazo. Dessa forma, optou-se pela realização deste trabalho, cujo objetivo foi avaliar a performance laboratorial de um

*Mestrandos do Curso de Ciências da Saúde, Área de Concentração Odontologia; **Professora Adjunta de Odontopediatria - Universidade de Brasília. 
FARIAS, D. G. de; AVELAR, R. P.; BEZERRA, A. C. B. Estudo comparativo da infiltração marginal em restaurações de classe V. Pesqui

Odontol Bras, v. 16, n. 1, p. 83-88, jan./mar. 2002.

sistema adesivo monocomponente - em que o "primer" e o adesivo se encontram no mesmo frasco, de um cimento de ionômero de vidro modificado por resina e de uma resina modificada por poliácidos, no selamento de margens gengivais de restaurações classe $\mathrm{V}$.

\section{MATERIAL E MÉTODO}

Foram utilizados vinte terceiros molares humanos íntegros extraídos, conservados em soro fisiológico, e submetidos a exames com lupa estereoscópica $(10 \mathrm{X})$, para verificação de possiveis trincas de esmalte ou defeitos estruturais.

As cavidades de classe $\mathrm{V}$ foram preparadas nas faces vestibular e lingual dos dentes, no limite amelocementário, em alta rotação, sob refrigeração ar/água, utilizando pontas diamantadas 1092 KG Sonresen, tendo um "stop" de resina adaptado para limitação da profundidade. As pontas diamantadas foram substituídas a cada 10 preparos $^{3}$.

Para padronização dos preparos, utilizou-se matriz metálica fenestrada como guia, com as seguintes dimensões: $4 \mathrm{~mm}$ para a distância mésio-distal, $3 \mathrm{~mm}$ para a distância cérvico-oclusal, e $2 \mathrm{~mm}$ de profundidade ${ }^{3}$. Todas as margens foram terminadas em $90^{\circ}$ com a superficie externa.

Os preparos concluídos foram mantidos úmidos com a presença de algodão embebido em água. Os espécimes foram aleatoriamente divididos considerando-se 4 grupos, e procedeu-se à restauração com diferentes materiais, seguindo-se as recomendações dos fabricantes (Quadro 1).

No grupo 1, o Vitremer Primer foi aplicado por 30 segundos, seguido de uma suave secagem com jato de ar e fotopolimerização (20 segundos). O cimento de ionômero de vidro modificado por resina Vitremer foi manipulado e inserido na cavidade

QUADRO 1 - Distribuição dos materiais restauradores nas cavidades preparadas.

\begin{tabular}{|c|c|c|c||}
\hline \hline Grupos & $\begin{array}{c}\mathrm{N}^{\mathrm{o}} \text { de } \\
\text { cavidades }\end{array}$ & Material & Fabricante \\
\hline $\begin{array}{c}\mathrm{G} 1 \\
\text { (Vestibular) }\end{array}$ & 10 & Vitremer & $3 \mathrm{M}$ \\
\hline $\begin{array}{c}\mathrm{G} 2 \\
\text { (Lingual) }\end{array}$ & 10 & $\begin{array}{c}\text { Vitremer + Syntac } \\
\text { Sprint/Tetric Ceram }\end{array}$ & $\begin{array}{c}3 \mathrm{M} / \\
\text { Vivadent }\end{array}$ \\
\hline $\begin{array}{c}\text { G3 } \\
\text { (Vestibular) }\end{array}$ & 10 & $\begin{array}{c}\text { Syntac Sprint/ } \\
\text { Tetric Ceram }\end{array}$ & Vivadent \\
\hline $\begin{array}{c}\text { G4 } \\
\text { (Lingual) }\end{array}$ & 10 & $\begin{array}{c}\text { Prime \& Bond 2.1/ } \\
\text { Variglass }\end{array}$ & Dentsply \\
\hline
\end{tabular}

com o auxílio de seringa tipo Centrix, em único bloco. O material foi fotopolimerizado por 40 segundos, sob compressão com uma fita de acetato de celulose.

No grupo 2 , foi realizada a técnica de sanduíche, utilizando-se a combinação de cimento ionomérico modificado por resina e sistema adesivo/ resina composta. O cimento de ionômero de vidro Vitremer foi aplicado na parede axial numa espessura minima de $0,5 \mathrm{~mm}$, seguindo-se os passos descritos para o grupo 1. Em seguida, foi realizado o condicionamento das margens de esmalte e da superficie dentinária exposta com o ácido fosfórico a $37 \%$ do sistema adesivo Syntac Sprint, por 15 segundos. O cimento não foi condicionado e, após lavagem da cavidade, o sistema adesivo foi aplicado com pincel, durante 20 segundos. Removeu-se o excesso com leve jato de ar, e seguiu-se à fotopolimerização por 20 segundos. Uma nova camada do sistema adesivo foi aplicada de modo semelhante. A resina composta Tetric Ceram foi inserida em camadas, com auxílio de espátula antiaderente, sendo o primeiro incremento em direção à parede oclusal, o segundo em direção à parede gengival e o terceiro restabelecendo o contorno anatômico do dente. Cada incremento foi polimerizado por 40 segundos.

No grupo 3, foi realizada a técnica de condicionamento ácido total e hibridização, utilizando-se o sistema adesivo Syntac Sprint e a resina composta Tetric Ceram. Toda superficie de esmalte e dentina do preparo foi condicionada com ácido fosfórico a $37 \%$ por 15 segundos, seguida da lavagem e secagem da cavidade sem desidratá-la. A aplicação do sistema adesivo e da resina composta foi semelhante à descrita para o grupo 2 .

Para as cavidades do grupo 4, utilizou-se a técnica de hibridização com o sistema adesivo Prime $\&$ Bond 2.1, seguida da restauração com uma resina modificada por poliácido - Variglass. O esmalte e a dentina do preparo foram condicionados com ácido fosfórico a $37 \%$ por 15 segundos, seguidos da lavagem e secagem da cavidade com suave jato de ar, sem desidratá-la. O sistema adesivo foi aplicado por 20 segundos, removido o excesso com leve jato de ar, e fotopolimerizado por 10 segundos. Uma segunda camada foi aplicada de maneira semelhante. O Variglass foi manipulado e inserido na cavidade em incrementos, com o auxílio de seringa Centrix. Cada camada foi polimerizada por 40 segundos.

Os espécimes foram armazenados em água, e todas as restaurações foram acabadas e polidas 
FARIAS, D. G. de; AVELAR, R. P.; BEZERRA, A. C. B. Estudo comparativo da infiltração marginal em restaurações de classe V. Pesqui Odontol Bras, v. 16, n. 1, p. 83-88, jan./mar. 2002.

com discos de acabamento Sof-Lex (3M) após 48 horas, exceto para o G1, para o qual o fabricante recomenda acabamento imediato.

Todos os dentes tiveram seus ápices selados com resina composta fotopolimerizável, e delimitada a área ao redor da restauração com $1 \mathrm{~mm}$ aquém da interface dente/restauração, procedendo-se então à pintura dos dentes com esmalte de unha incolor, em três camadas, com intervalos de aplicação até secagem completa ${ }^{3}$.

Para verificação da infiltração marginal, os espécimes foram submetidos a um tratamento térmico, imersos em solução de azul de metileno a $0,5 \%$, constituído de 5 ciclos de 9 minutos, sendo 3 minutos a $5^{\circ} \pm 2^{\circ} \mathrm{C}, 3$ minutos a $37^{\circ} \pm 2^{\circ} \mathrm{C}$, e 3 minutos a $55^{\circ} \pm 2^{\circ} \mathrm{C}$, perfazendo um total de $45 \mathrm{mi}-$ nutos, durante o qual os corpos-de-prova foram introduzidos num sistema de rodízio. Após termociclagem, os dentes foram lavados em água corrente, durante 10 minutos, para remoção do corante excedente ${ }^{7}$.

Em seguida, os dentes foram seccionados no sentido vestíbulo-lingual, através do centro da restauração, utilizando-se discos diamantados refrigerados, e submetidos a análise do grau de infiltração ao longo da parede gengival, com auxílio de lupa estereoscópica $(40 \mathrm{X})$. Dois examinadores calibrados determinaram os escores baseados no grau de penetração do corante, adotando o critério de RETIEF et al. ${ }^{10}$ (1982), e em caso de discordância, um consenso era obtido pelos mesmos.

- Grau 0 - Nenhuma penetração do corante;

- Grau 1 - Penetração do corante em esmalte;

- Grau 2 - Penetração do corante em dentina;

- Grau 3 - Penetração do corante ao longo da parede axial, com conseqüente penetração nos túbulos dentinários;

- Grau 4 - Penetração do corante ao longo da parede axial, em direção à câmara pulpar.

\section{RESULTADOS}

Os resultados obtidos na avaliação da infiltração marginal estão contidos nas Tabelas 1 e 2, e no Gráfico 1.

O teste não-paramétrico de Mann-Whitney foi empregado para verificar diferenças na distribuição do grau de microinfiltração, tendo sido os grupos comparados dois a dois pela média dos postos. O teste revelou a existência de diferença estatística entre os grupos 1 e 4 , a nível de $5 \%(\alpha=0,05)$, mas não foram demonstradas diferenças estatistica-
TABELA 1 - Freqüência dos escores de infiltração marginal observados na parede cervical, em diferentes condições experimentais.

\begin{tabular}{c|c|c|c|c|c|c}
\hline \multirow{2}{*}{ Grupos } & \multirow{2}{*}{ Material } & \multicolumn{5}{|c}{ Escores } \\
\cline { 3 - 7 } & Vitremer & 9 & 1 & 0 & 0 & 0 \\
\hline G1 & $\begin{array}{c}\text { Vitremer + Syntac } \\
\text { Sprint/Tetric Ceram }\end{array}$ & 7 & 2 & 1 & 0 & 0 \\
\hline G3 & $\begin{array}{c}\text { Syntac Sprint/ } \\
\text { Tetric Ceram }\end{array}$ & 6 & 3 & 0 & 0 & 1 \\
\hline G4 & $\begin{array}{c}\text { Prime \& Bond 2.1/ } \\
\text { Variglass }\end{array}$ & 4 & 5 & 1 & 0 & 0 \\
\hline \hline
\end{tabular}

TABELA 2 - Escores médios de infiltração marginal na parede gengival em diferentes condições experimentais.

\begin{tabular}{c|c}
\hline \hline Grupos & Escores médios \\
\hline G1 & 0,10 \\
\hline G2 & 0,40 \\
\hline G3 & 0,70 \\
\hline G4 & 0,70 \\
\hline \hline
\end{tabular}

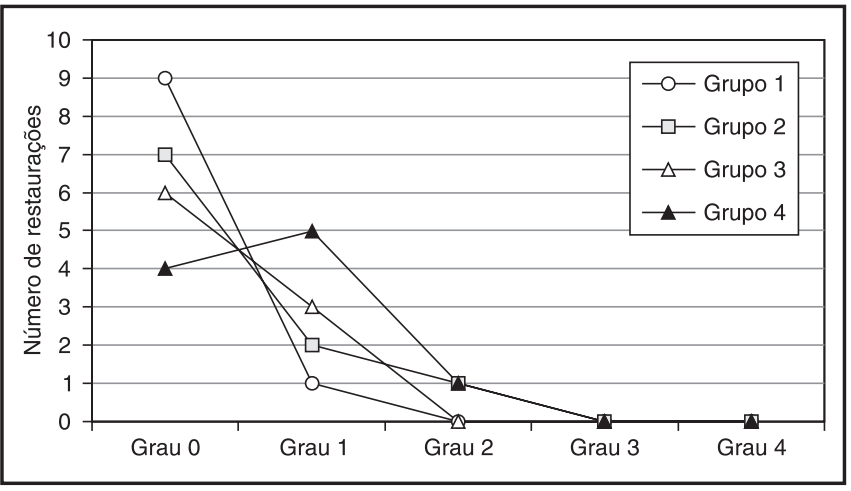

GRÁFICO 1 - Freqüência dos escores de infiltração marginal observados na parede cervical, em diferentes condições experimentais.

mente significantes entre os demais grupos (Tabelas 3 a 8).

\section{DISCUSSÃO}

Com base nos resultados, verifica-se que, apesar do bom desempenho laboratorial, nenhum material foi capaz de eliminar totalmente a infiltração marginal na margem gengival, concordando com trabalhos semelhantes anteriormente publi$\operatorname{cados}^{3,4,5,8,9,11,12}$.

Demonstrou-se que tanto o material Vitremer 
FARIAS, D. G. de; AVELAR, R. P.; BEZERRA, A. C. B. Estudo comparativo da infiltração marginal em restaurações de classe V. Pesqui

Odontol Bras, v. 16, n. 1, p. 83-88, jan./mar. 2002.

TABELA 3 - Análise estatística de Mann-Whitney, para comparação entre os grupos 1 e 4 .

\begin{tabular}{l|c}
\hline \hline Valor de U(1) & 20 \\
\hline Valor de U(2) & 80 \\
\hline Valor calculado de “z” & $-2,2678$ \\
\hline Probabilidade de igualdade & $1,17 \%$ \\
\hline
\end{tabular}

Significante ao nível de $5 \%(\alpha=0,05)$

TABELA 4 - Análise estatística de Mann-Whitney, para comparação entre os grupos 1 e 2 .

\begin{tabular}{l|l}
\hline \hline Valor de U(1) & 39,5 \\
\hline Valor de U(2) & 60,5 \\
\hline Valor calculado de “z” & $-0,7937$ \\
\hline Probabilidade de igualdade & $21,37 \%$ \\
\hline
\end{tabular}

Não-significante, amostras iguais $(\alpha>0,05)$

TABELA 5 - Análise estatística de Mann-Whitney, para comparação entre os grupos 1 e 3 .

\begin{tabular}{l|c}
\hline \hline Valor de U(1) & 34,5 \\
\hline Valor de U(2) & 56,5 \\
\hline Valor calculado de “z” & $-1,1717$ \\
\hline Probabilidade de igualdade & $12,07 \%$ \\
\hline \multicolumn{2}{l}{ Não-significante, amostras iguais $(\alpha>0,05)$} \\
\hline \hline
\end{tabular}

(3M) como o Variglass (Dentsply) não foram capazes de impedir totalmente a microinfiltração na parede gengival, embora o primeiro tenha permitido uma menor penetração do corante, sob o ponto de vista estatístico, quando comparado com o Variglass.

BRACKETT et al. ${ }^{4}$ (1998) e CARRARA et $a .^{5}$ (1997) analisando a infiltração marginal de cimentos ionoméricos modificados por resina e resinas compostas modificadas por poliácidos, também obtiveram resultados semelhantes com esses materiais, concluindo que as resinas compostas modificadas por poliácidos não devem ser indicadas como um substituto dos cimentos ionoméricos, frente ao grau de infiltração que ainda permitem.

A evolução dos cimentos ionoméricos convencionais levou à introdução dos cimentos ionoméricos modificados por resina e das resinas compostas modificadas por poliácidos, que apresentam algumas vantagens sobre os primeiros, como
TABELA 6 - Análise estatística de Mann-Whitney, para comparação entre os grupos 2 e 3 .

\begin{tabular}{l|c}
\hline \hline Valor de U(1) & 45 \\
\hline Valor de U(2) & 55 \\
\hline Valor calculado de "z" & $-0,3780$ \\
\hline Probabilidade de igualdade & $35,27 \%$ \\
\hline \multicolumn{2}{l}{ Não-significante, amostras iguais $(\alpha>0,05)$} \\
\hline
\end{tabular}

TABELA 7 - Análise estatística de Mann-Whitney, para comparação entre os grupos 2 e 4 .

\begin{tabular}{l|c}
\hline \hline Valor de U(1) & 36,5 \\
\hline Valor de U(2) & 63,5 \\
\hline Valor calculado de “z” & $-1,0205$ \\
\hline Probabilidade de igualdade & $15,37 \%$ \\
\hline \multicolumn{2}{l}{ Não-significante, amostras iguais $(\alpha>0,05)$} \\
\hline \hline
\end{tabular}

TABELA 8 - Análise estatística de Mann-Whitney, para comparação entre os grupos 3 e 4 .

\begin{tabular}{l|c}
\hline \hline Valor de U(1) & 41,5 \\
\hline Valor de U(2) & 58,5 \\
\hline Valor calculado de "z" & $-0,6425$ \\
\hline Probabilidade de igualdade & $26,03 \%$ \\
\hline \multicolumn{2}{l}{ Não-significante, amostras iguais $(\alpha>0,05)$} \\
\hline \hline
\end{tabular}

melhor aparência, resistência e polimento imediato $^{4,5,13}$. Entretanto, a diferente composição desses materiais leva a um comportamento laboratorial e clínico distintos.

Para os cimentos ionoméricos modificados por resina, a reação ácido-básica típica dos cimentos convencionais é suplementada por um segundo processo de reação química, fotoativada ${ }^{4,13}$. A fórmula atual desses cimentos varia de acordo com as marcas comerciais do produto, mas seu conteúdo de resina deve ser entre 4,5 e $6,0 \%$ após a presa do material ${ }^{5}$.

Para as resinas compostas modificadas por poliácido, o conteúdo de resina é bastante elevado, e o material não apresenta a reação de autogeleificação ácido-básica dos cimentos ionoméricos convencionais, mas apenas uma reação de presa desencadeada na presença de luz halógena ${ }^{5}$.

De acordo com CARRARA et al. ${ }^{5}$ (1997), os altos valores de infiltração observados para o Variglass 
FARIAS, D. G. de; AVELAR, R. P.; BEZERRA, A. C. B. Estudo comparativo da infiltração marginal em restaurações de classe V. Pesqui Odontol Bras, v. 16, n. 1, p. 83-88, jan./mar. 2002.

(Dentsply) talvez possam ser explicados pelo fato de o material possuir uma alta porcentagem de monômeros que lhe confere uma elevada contração de polimerização e um módulo de expansão térmica elevado.

Para as restaurações com o material Variglass, o condicionamento ácido das estruturas dentárias, seguido da aplicação de adesivos dentinários - técnica de hibridização - é opcional, de acordo com as instruções do fabricante. Embora HOANG et $a .^{6}{ }^{6}$ (1996) tenham demonstrado um melhor vedamento marginal nas restaurações com resinas modificadas por poliácido quando a técnica de hibridização foi associada, neste estudo, o uso da técnica de hibridização através de sistema adesivo Prime \& Bond 2.1 (Dentsply) não pareceu ser efetivo na prevenção de microinfiltração.

A aplicação de um sistema adesivo hidrofilico constitui uma alternativa para o vedamento marginal, mas estudos demonstram que seu emprego ainda não é capaz de conferir um selamento hermético e de eliminar a microinfiltração, mas sim de minimizá-la, dependendo do tipo de material restaurador empregado ${ }^{6,7,8,12,13,14}$. Neste estudo, não foram observadas diferenças estatísticas quando o uso de sistema adesivo/resina composta foi comparado com o uso de sistema adesivo/resina modi- ficada por poliácido e cimento ionomérico modificado por resina, o que indica que os materiais não apresentaram um comportamento laboratorial discrepante entre si, mas permitiram um grau de microinfiltração discreto, localizado na grande maioria, apenas em esmalte (Tabelas 1 e 2; Gráfico 1).

A despeito do grande número de trabalhos científicos desenvolvidos sobre microinfiltração marginal sob restaurações estéticas, novos estudos são necessários para se obter maiores informações sobre os materiais que possuem o melhor desempenho para a clínica moderna, principalmente naquelas situações em que as margens do preparo cavitário se encontram localizadas em dentina.

\section{CONCLUSÃO}

Frente às condições desse trabalho, pode-se concluir que o cimento ionomérico modificado por resina Vitremer $(3 \mathrm{M})$ proporcionou melhor selamento para a margem gengival em restaurações classe V, quando comparado com o sistema adesivo Prime \& Bond 2.1 associado com a resina composta modificada por poliácido Variglass (Dentsply).

FARIAS, D. G. de; AVELAR, R. P.; BEZERRA, A. C. B. Comparative study of microleakage in class V restorations. Pesqui Odontol Bras, v. 16, n. 1, p. 83-88, jan./mar. 2002.

The aim of this study was to analyze the laboratorial performance of dentin-bonding agents, glass ionomer cements and compomers, regarding the sealing of the gingival margins of class $\mathrm{V}$ cavities, prepared on the cementoenamel junction. Standardized class V cavities $(4 \times 3 \times 2 \mathrm{~mm})$ were prepared on the buccal and lingual/palatal surfaces of 20 extracted human third molars. Forty cavities were restored with different combinations of materials, as follows: group 1 (G1)- Vitremer (3M); group 2 (G2)- Vitremer (3M) and Syntac Sprint/Tetric Ceram (Vivadent); Group 3 (G3)- Syntac Sprint/Tetric Ceram (Vivadent); Group 4 (G4)- Prime \& Bond 2.1/Variglass (Dentsply). After thermocycling in methylene blue dye, the specimens were longitudinally sectioned and analyzed with a stereoscopic lens in order to evaluate the leakage on the gingival margin. The obtained data were analyzed with the Mann-Whitney test, and the results revealed significantly better sealing $(\mathrm{p}=0.05)$ in $\mathrm{G} 1$, when compared with $\mathrm{G} 4$. No significant differences were seen between the other groups. Under the given conditions, Vitremer offered better sealing of the gingival margin than the system Prime \& Bond 2.1/Variglass.

UNITERMS: Dental leakage; Dentin-bonding agents; Glass ionomer cements; Composite resins.

\section{REFERÊNCIAS BIBLIOGRÁFICAS}

1. ALBERTON, S. B. et al. União entre resina composta e cimento de ionômero de vidro. Odonto 10. Caderno Documento, v. 2, n. 4, p. 290-293, 1991.

2. ARAÚJO, M. A. M. et al. Restauração de classe V. Avaliação da infiltração marginal com diferentes procedimentos e materiais. Rev Odontol UNESP, v. 22, n. 2, p. 239-247, 1993.

3. ARAÚJO, M. A. M. et al. Avaliação da infiltração marginal na interface resina composta-cimento de ionômero de vidro e deste com a dentina. Rev Odontol UNESP, v. 24, n. 1, p. 69-78, 1995.

4. BRACKETT, W. W. et al. Microleakage of class V compomer and light-cured glass ionomer restorations. J Prosthet Dent, v. 79, n. 3, p. 261-263, 1998.

5. CARRARA, C. E. et al. Infiltração marginal de cimentos ionoméricos modificados por resina. Rev Odontol USP, v. 11, n. 2, p. 75-78, 1997. 
FARIAS, D. G. de; AVELAR, R. P.; BEZERRA, A. C. B. Estudo comparativo da infiltração marginal em restaurações de classe V. Pesqui

Odontol Bras, v. 16, n. 1, p. 83-88, jan./mar. 2002.

6. HOANG, E. et al. Comparison of microleakage using etched and non etched techniques in class $V$ restorations: an in vitro study. J Dent Res, v. 75, p. 388, 1996. [Abstract 2,966].

7. MELLO, J. B. et al. A influência do condicionamento ácido da dentina no selamento marginal de um adesivo. Rev Odontol UNESP, v. 25, n. 1, p. 69-79, 1996.

8. PIMENTA, L. A.; PAIVA, O. C. Efetividade de adesivos dentinários hidrofilicos no controle da microinfiltração marginal. Rev Assoc Paul Cir Dent, v. 51, n. 2, p. 183-187, 1997.

9. PORTO NETO, S. T. et al. Estudo comparativo da infiltração marginal em cavidades de classe $\mathrm{V}$ restauradas com cimento de ionômero de vidro. Rev Odontol UNESP, v. 23, n. 1, p. 91-99, 1994.
10. RETIEF, D. H. et al. Effect of cavo surface treatment on marginal leakage in class $\mathrm{V}$ composite resin restorations. J Prosthet Dent, v. 47, p. 496-501, 1982.

11. SJÖDIN, M. U.; DIJKEN, J. V. Resin modified glass ionomer cements. In vitro microleakage in direct class $\mathrm{V}$ and class II sandwich restorations. Swed Dent J, v. 20, p. 77-86, 1996.

12. SWIFT, E. J.; VALLEY, B. D. Microleakage of etched-dentin composite resin restorations. Quintessence Int, v. 23, n. 7, p. 505-508, 1992.

13. TRUSHKOWSKY, R. D.; GWINNETT, A. J. Microleakage of class $\mathrm{V}$ composite, resin sandwich, and resin-modified glass ionomers. Am J Dent, v. 9, p. 96-99, 1996.

14. TYAS, M. J. Clinical evaluation of five adhesive systems: three-year results. Int Dent J, v. 46, p. 10-14, 1996.

Recebido para publicação em 08/10/99 Enviado para reformulação em 24/04/00 Aceito para publicação em 19/07/01

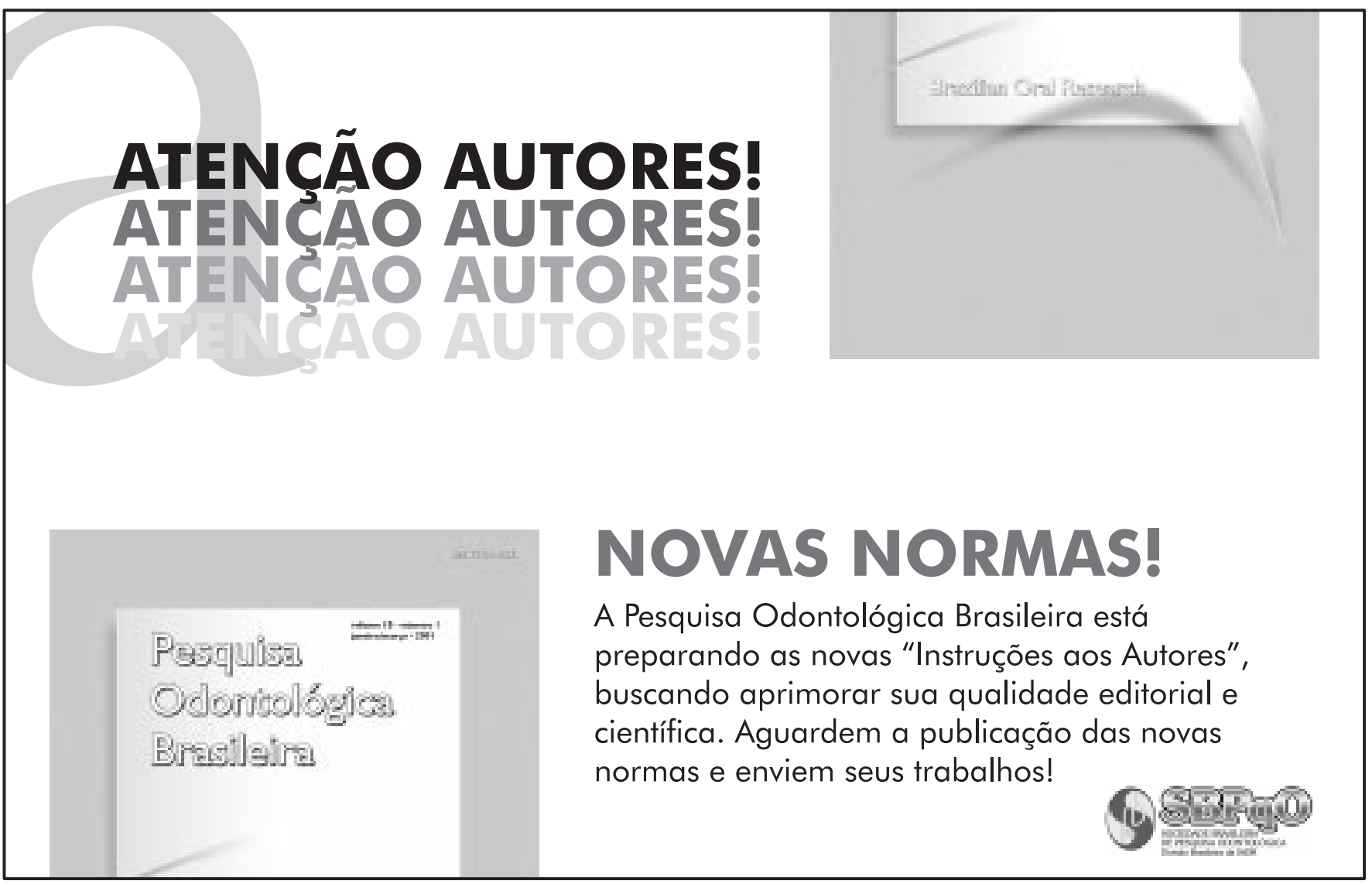

